\title{
Reconstrucción craneofacial compleja: malla de titanio, hueso autólogo preservado en óxido de etileno y reconstrucciones tridimensionales en polimetilmetacrilato (HTR-PMI) \\ Complex craneofacial reconstruction: titanium mesh, autologous hone preserved in ethylene oxide and tridimensional polimetilmetacrilate implants (HTR-PMI)
}

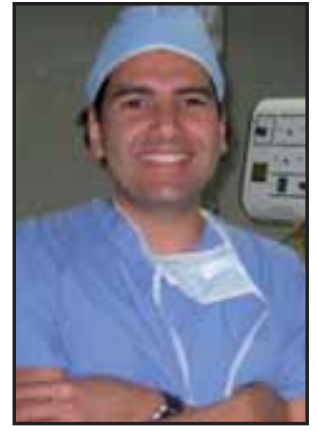

Flores-Lima, G.

Flores-Lima, G.*, Lovo Iglesias, E.**

\section{Resumen}

La evolución de la Cirugía Craneofacial se inicio con Jean Paul Tessier, quien en 1967 preconizó el uso de injertos autólogos de hueso fresco en gran cantidad para cubrir extensas brechas óseas en la corrección de disóstosis craneofacial. Recientemente, diferentes tipos de reconstrucción utilizando hueso autólogo preservado en oxido de etileno y materiales como el polimetilmetacrilato poroso confeccionado a medida, han permitido también la corrección de grandes defectos óseos craneofaciales. Presentamos nuestra experiencia inicial en el uso de estas técnicas a través de un análisis retrospectivo sobre 21 pacientes operados por un equipo multidisciplinario entre Enero del 2007 y Marzo del 2009 en el Hospital Militar, Centro Panamericano de Ojos y Hospital de Diagnóstico de El Salvador, en los que se utilizaron formas alternativas pera reconstrucción de calota craneana, piso de órbita, fosa craneal anterior, área órbito-cigomática y maxilar superior.

No registramos casos de infección o retirada de material de osteosíntesis aloplástico o de los injertos autólogos, ni hubo fístulas. Si se presentó una úlcera postraumática en una zona de unión de tejido desvitalizado, que se resolvió con tratamiento conservador. Los resultados estéticos obtenidos fueron de aceptables a buenos.

Como conclusión, el equipo multidisciplinario, la combinación de técnicas quirúrgicas y el uso de material protésico para la reconstrucción craneofacial compleja ha dado como resultado avances significativos desde el punto de vista funcional y estético ante lesiones que involucran esta compleja área anatómica.

\section{Palabras clave Trauma Craneofacial, Craneocerebral, Titanio, Óxido de Etileno, Polimetilme- tacrilato.}

Código numérico 2024-24-2432

\footnotetext{
* Cirujano Plástico.

* J efe del Servicio de Neurocirugía.

Hospital Militar Central. El Salvador, San Salvador, Centroamérica
}

\section{Abstract}

The evolution of craniofacial surgery began with Jean Paul Tessier, who in 1967 supported the use of fresh autologous bone to cover bone defects in the craniofacial area. Recently different types of reconstructions using autologous bone preserved in ethylene oxide and advanced custom-made polimetacrilate implants that have allowed more complex and esthetically rewarding procedures to patients have suffered extensive bone loss in the craniofacial area. We report our initial experience using these techniques with a retrospective analysis of 21 patients operated from January 2007 to March 2009 by a multidisciplinary team in the Hospital Militar, Centro Panamericano de Ojos and Hospital de Diagnóstico of El Salvador, who required complex craneofacial reconstruction using one or more techniques to cover the defects. The reconstructed areas have been cranium, orbital roof and floor as well as cigomatic region, anterior cranium fossa and superior maxillary sinuous.

No infections or retrieval of material for reconstruction or fistulas were reported and only one post-traumatic ulcer that resolved in a conservative way. The esthetic results in the patients operated have been considered acceptable, to excellent.

As a conclusions, multidisciplinary team, combination of surgical techniques and the acquisition of advanced prosthesis material for craniofacial reconstructions has resulted in a significant advance from the functional and aesthetic point of view in areas that involve this anatomically complex area.

$\begin{array}{ll}\text { Key words } & \text { Craniofacial trauma, Craneocerebral, } \\ & \text { Titanium, Ethylene oxide, } \\ & \text { Polyhydroxyethylmethacrylate. } \\ \text { Numeral Gode } & 2024-24-2432\end{array}$




\section{Introducción}

La región cráneo-facial representa un reto desde el punto de vista reconstructivo. Anatómicamente está relacionada con cavidades no estériles (fosas nasales, cavidad oral y senos paranasales) con potencial contacto por cercanía con áreas sumamente vitales (cerebro). Además, desde el punto de vista estético es la carta de presentación del ser humano, lo cual puede conllevar impedimentos sociales o psicológicos cuando existen desfiguramientos debidos a traumas o tumores.

Desde 1951, cuando Leventhal (1) realizó sus estudios en conejos y ratas introduciendo fragmentos de titanio, concluyó con la interrogante: "espero que en el futuro, se fabriquen placas y tornillos de este material", se ha evolucionado mucho. Probablemente esta evolución se pueda atribuir a la propiedad inerte clásica del titanio y a su dureza; pero muy poco hubiera evolucionado la Cirugía Craneofacial de no haber sido por Jean Paul Tessier (2), que en 1967, con sus visionarios estudios, preconizó la utilización de injertos autólogos de hueso fresco en grandes cantidades para cubrir extensas brechas óseas en la corrección de disóstosis craneofaciales. Branemark en 1977 (3), un pionero en el área de la implantología oral, describió
Tabla I: Características de las lesiones en el grupo de pacientes.

\begin{tabular}{lr}
\hline Causa de la lesión & N. \\
\hline Tumores & 6 \\
Accidente cerebrovascular masivo & 1 \\
Heridas por arma de fuego & 4 \\
Accidentes de tránsito & 2 \\
Traumatismo por puño u objeto & 8 \\
Total de pacientes & 21 \\
& \\
Regiones anatómicas tratadas & \\
Calota craneana & 8 \\
Piso de órbita & 12 \\
Maxilar superior & 2 \\
Área órbito-cigomática & 2 \\
Fosa craneal anterior & 1 \\
Total de regiones tratadas & 25 \\
& \\
Tipos de Abordajes & \\
Transconjuntival más cantotomía lateral & 12 \\
Vestibular & 2 \\
Fronto-órbito-cigomático & 2 \\
Bicoronal & 4 \\
Colgajo de Trauma & 5 \\
\hline
\end{tabular}

su experiencia con implantes de titanio en pacientes edéntulos obteniendo excelente resultados, lo que fue

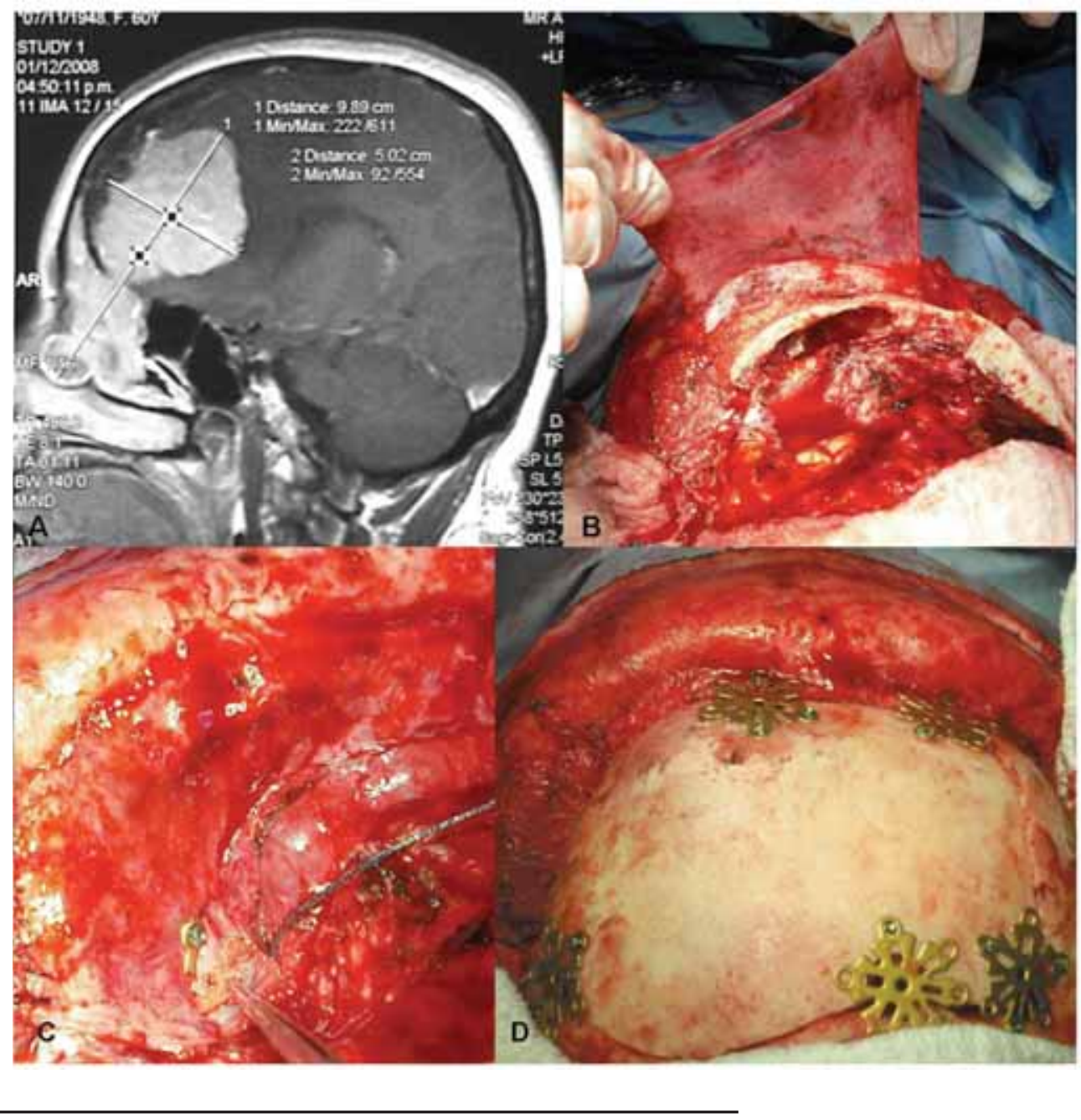

Fig. 1. A, Resonancia magnética T1 sagital que muestra tumor cráneo facial extenso que involucra bóveda craneana, senos paranasales y cavidad nasal. B, Colgajo de pericráneo para reconstrucción de la fosa craneana anterior (postresección del tumor). C, Colgajo de pericráneo fijado a techo orbitario con miniplacas de titanio y tornillos autoroscables. D, Cierre del cráneo con cubretrépanos. 


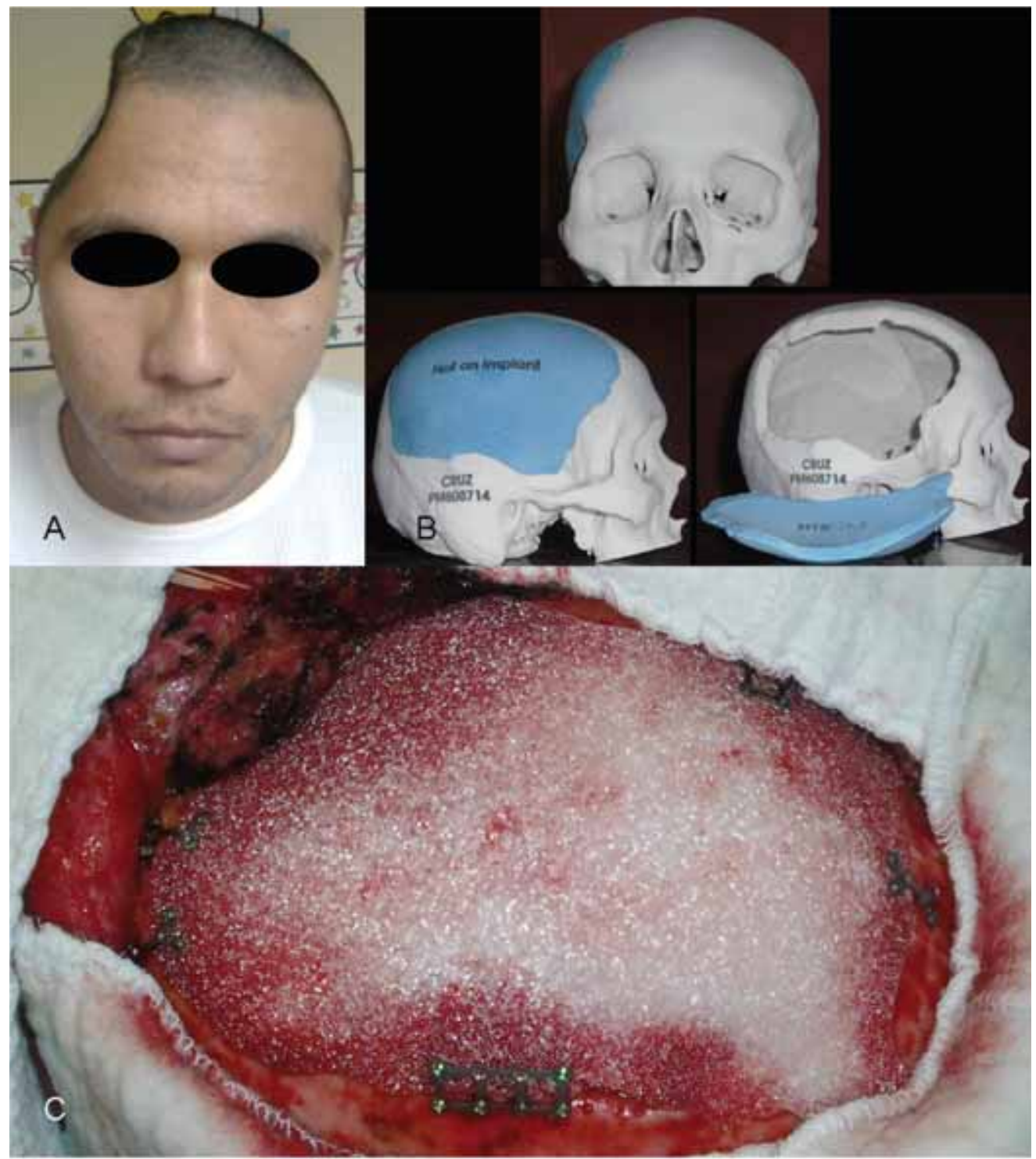

Fig. 2. A, Vista frontal de defecto craneano derecho. B, Modelo reconstruido de prueba que muestra (celeste) el tamaño real del implante a colocar. C, Implante (HTR-PMI) definitivo fijado a los bordes del cráneo con placas de titanio.

un paso determinante para el inicio de la confección de placas y tornillos de titanio. Más recientemente diferentes tipos de reconstrucción han empleado hueso autólogo preservado en óxido de etileno (4) y materiales como el polimetilmetacrilato poroso (5) confeccionado a medida para reconstrucción tridimensional (3-D) con Tomografía computarizada (TC) para la corrección de grandes defectos óseos, que proporcionan armas más avanzadas para corregir la presencia de amplios defectos óseos cráneo-faciales y la morbimortalidad que éstos conllevan.

En este artículo presentamos nuestra experiencia inicial utilizando estas técnicas de preservación y reconstrucción para el tratamiento de defectos craneofaciales complejos que involucran tanto a Cirugía Plástica como a Neurocirugía.

\section{Material y método}

Realizamos un análisis retrospectivo sobre 21 pacientes operados en el Hospital Militar, Centro Pan- americano de Ojos y Hospital de Diagnóstico de El Salvador, entre Enero del 2007 y Marzo del 2009, en los que se utilizaron formas alternativas pera reconstrucción de calota craneana, piso de órbita, fosa craneal anterior, área orbito-cigomática y maxilar superior.

De estos 21 pacientes, 19 (90.4\%) fueron hombres y $2(9.6 \%)$ mujeres, entre los 12 y los 90 años de edad, con una media de 39 años. Las causas de los defectos fueron: $6(29 \%)$ tumores (2 meningiomas petroclivales, 1 osteoma osteoide, 1 tumor dermoide de línea media y 2 meningiomas invasores), 1 (4.6\%) accidente cerebrovascular (craneotomía descompresiva), 4 (19\%) heridas por arma de fuego, 2 (9.4\%) accidentes de tránsito y $8(38 \%)$ traumatismos contundentes.

Los sitios anatómicos reconstruidos fueron: 12 pisos de órbita, 2 maxilares superiores, 2 áreas órbitocigomáticas, 1 fosa anterior de cráneo y 8 calotas craneanas. Los abordajes realizados fueron: 12 transconjuntivales con cantotomía lateral, 2 vestibulares, 2 abordajes fronto-órbito-cigomáticos, 4 bicoronales y 5 colgajos de trauma (Tabla I). 


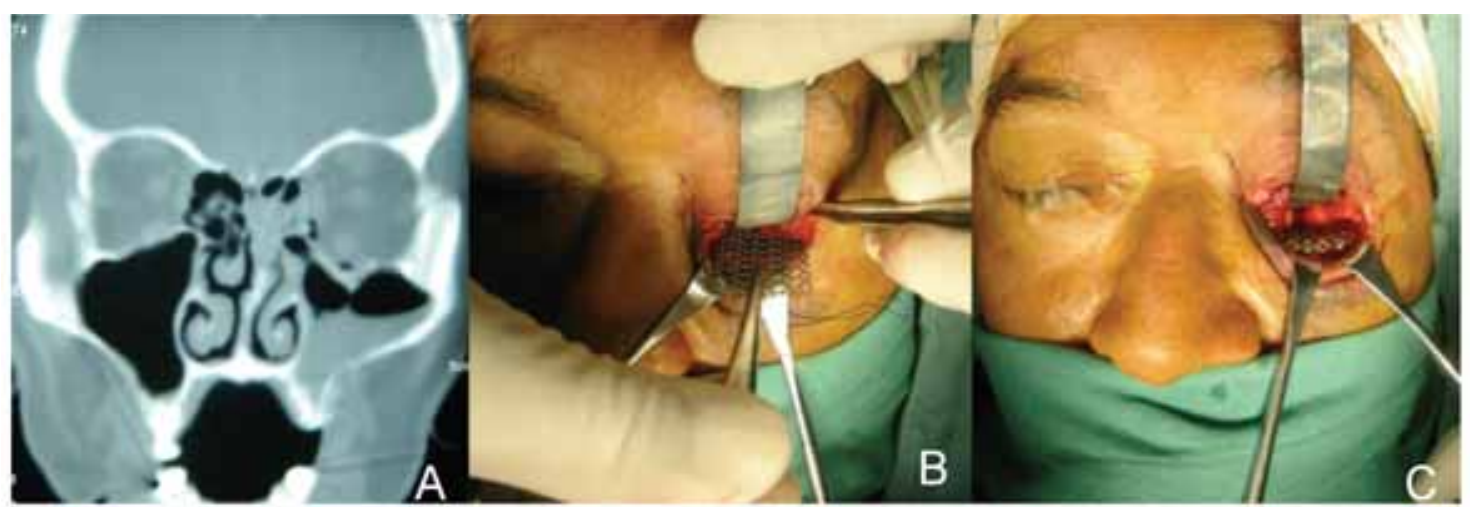

Fig. 3. A, TAC corte coronal que muestra fractura de piso de orbita. B, Abordaje transconjuntival con cantotomía lateral para introducción de malla. C, Fijación de malla con tornillos autorroscables.

Antes de la cirugía los pacientes fueron estudiados con Tomografía Computerizada (TC), con cortes coronales y axiales a intervalos que variaron entre 1$5 \mathrm{~mm}$ de espesor. El tipo de anestesia empleado para las cirugías fue general orotraqueal en todos los casos, exceptuando los 2 casos de reconstrucción de maxilar superior en los que la intubación fue nasotraqueal.

En los casos de reconstrucción de calota craneana, se utilizó cubretrépano de titanio de $22 \mathrm{~mm}$ de diámetro por $0.5 \mathrm{~mm}$ grosor, fijado con tornillos autorroscables de $1.5 \mathrm{~mm}$ por $5 \mathrm{~mm}$ de longitud (todos los tornillos autorroscables fueron de las mismas características). En el caso del meningioma invasor a nivel de fosa anterior de cráneo, el colgajo de pericráneo elevado fue fijado a la fosa anterior (techo orbitario) con malla de titanio de $0.6 \mathrm{~mm}$ de grosor fijado con tornillos autorroscables (Fig. 1). En los casos de reconstrucción órbito-cigomática, empleamos placa lineal de $0.5 \mathrm{~mm}$ de espesor. Para el resto de los casos, se utilizó el panel de cuadriculado regular de $0.6 \mathrm{~mm}$ de grosor fijado con tornillos autorroscables que iniciaban su fijación en los puntos cardinales de la malla y luego entre ellos; la cantidad mínima empleada fue de 7 tornillos y la máxima de 13 tornillos. En un caso se utilizó el sistema HTR-PMI, fijado con 3 placas tipo matriz del sistema $1.5 \mathrm{~mm}$ y 2 placas tipo $\mathrm{Y}$ con tornillos de $5 \mathrm{~mm}$ para la fijación (Fig. 2). Para las reconstrucciones de piso de órbita y pared medial
(Fig. 3), se realizó abordaje transconjuntival con cantotomía lateral en todos los casos, utilizando malla de titanio de $0.6 \mathrm{~mm}$ de grosor, confeccionada a la medida del defecto utilizando film plástico como molde antes de ser introducida en el piso y/o pared medial. Posteriormente se fijó con 3 tornillos autorroscables. En todos los casos se cerró la conjuntiva con puntos invertidos de 6-0 absorbibles. Para la reconstrucción del maxilar superior se realizó abordaje vestibular en ambos casos y se utilizó malla de titanio del panel de cuadriculado regular fijado con tornillos autorroscables y cierre en dos planos de mucosa oral con sutura absorbible; no se colocó fijación intermaxilar. En los casos de utilización de hueso autólogo preservado en oxido de etileno (7 casos) éste fue fijado con malla de titanio de panel de cuadriculado regular en ambos extremos utilizando tornillos autorroscables (Fig. 4) o cubretrépanos.

Se utilizó irrigación con solución salina 500cc más 1 ampolla de gentamicina en el intraoperatorio en todos los casos, así como antibiótico endovenoso profiláctico (Cefalosporina de tercera generación).

Resultados

No se registraron casos de muerte, procesos infecciosos, fístulas, ni hubo necesidad de retirar el material de osteosíntesis aloplástico o injerto autólogo utilizado.

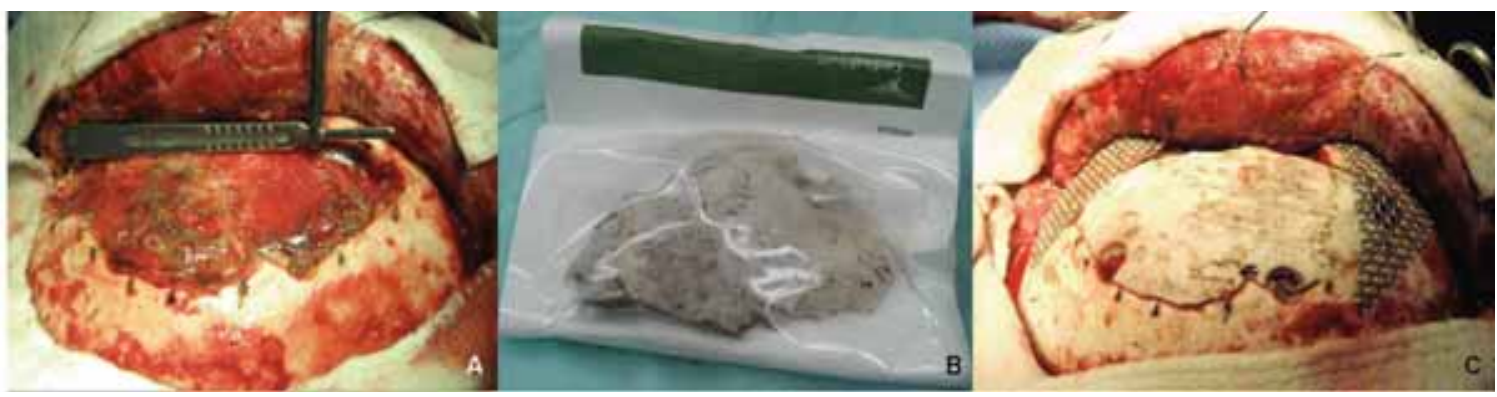

Fig. 4. A, Mango de bisturí utilizado como punto de referencia para ejemplificar la magnitud del defecto óseo (craneotomía descompresiva por trauma). B, Fragmento óseo esterilizado en óxido de etileno durante el primer tiempo quirúrgico. C, Colocación del fragmento óseo y complementación de las áreas de craniectomía, con mallas de titanio. 
Tuvimos una úlcera postraumática en un punto de unión de tejido desvitalizado, que se resolvió con tratamiento conservador.

El tiempo de seguimiento de los pacientes ha sido de 7 meses como media (de 3 a 22 meses). El tiempo medio operatorio fue de 2.45 horas (1.35 a 10.35 horas) y la estancia intrahospitalaria fue de 7 días como media (1 a 47 días).

\section{Discusión}

La reconstrucción de la región craneofacial debe ser realizada idealmente bajo una visión multidisciplinaria; las lesiones de esta zona son de diferentes especialistas. En este artículo inicial presentamos los casos que han sido trabajados en conjunto entre dos especialidades complementarias, Neurocirugía y Cirugía Plástica. Bajo este enfoque hemos desarrollado el proceso quirúrgico y hemos aportado la fortaleza individual de dos especialidades que han resultado sinérgicas en quirófano.
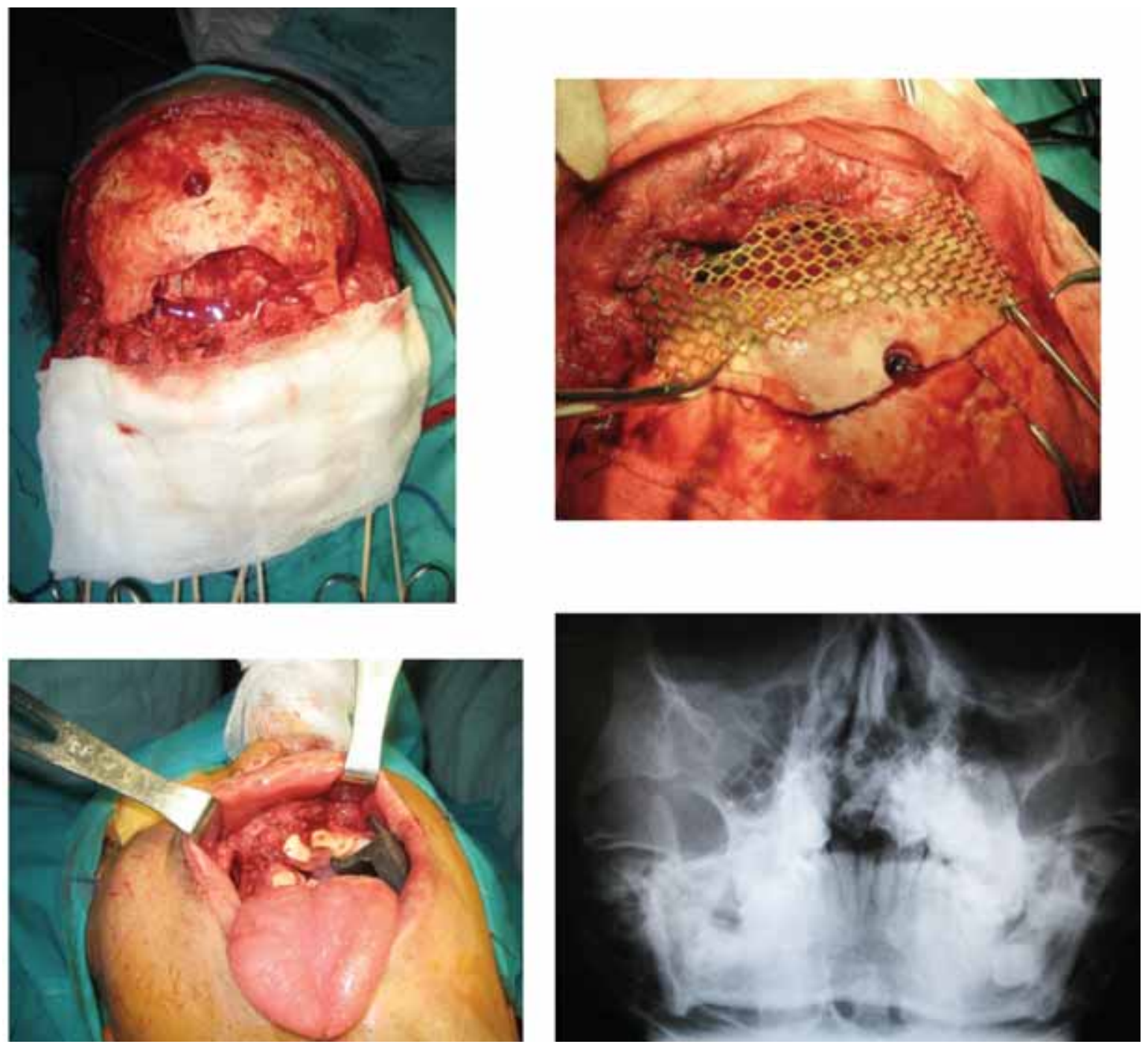

Fig. 5. A, Lesión de $9 \times 5 \mathrm{~cm}$. en área de hueso frontal, marcos orbitarios y nasion. B, Defecto cubierto con malla de titanio y asegurado con tornillos autorroscables. C, Fístula dentoalveolar corregida con colgajos locales. D, Rx en proyección frontal donde se observa la malla de titanio que cruza de maxilar derecho a maxilar izquierdo sobre la fístula corregida. de titanio fue expuesta a un ambiente hostil, el más representativo fue el tratamiento de una fractura frontal con afectación del seno paranasal y una segunda fractura tipo Leffort II en un paciente con labio leporino y paladar hendido, en el que su trazo de fractura cruzaba el sitio de la fístula dentoalveolar. Este caso es peculiar ya que ilustra la complejidad que puede requerir una reconstrucción en la que se involucran la cavidad craneana, los senos paranasales, la fosa nasal y la cavidad oral. La cirugía requirió dos tiempos; en el primero el equipo neuroquirúrgico evacuó hematomas epidurales frontales, cerró las laceraciones durales, extrajo los fragmentos óseos alojados en el seno frontal y en la cavidad nasal, reconstruyó la fosa anterior del cráneo utilizando un colgajo de pericráneo que fijaron a la duramadre y posteriormente, Cirugía Plástica realizó la reconstrucción ósea con titanio y en un segundo tiempo, el cierre de la fístula dentoalveolar y sobre la fístula ya cerrada, colocó una malla de titanio que cruzaba de maxilar derecho a maxilar izquierdo (Fig. 5).
Dentro de los casos combinados en los que la malla

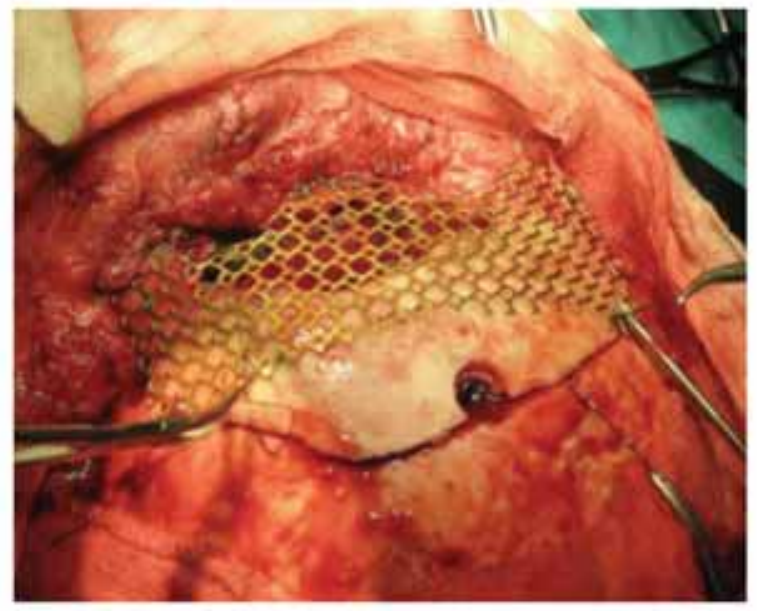


La malla de titanio es considerada como un material aloplástico con muy buenos resultados en cuestión de integración con el epitelio, inclusive cuando se utiliza en senos paranasales y áreas nasales, cavidad oral o faríngea. Esta malla es incorporada por epitelio de transición y células glandulares en cuestión de 3 meses (6), lo que sirve de ejemplo de la bondad del material.

En los hospitales participantes en nuestro trabajo en los que se realizó la investigación y se practica la Neurocirugía (Hospital Militar y Hospital de Diagnóstico en El Salvador), se carece de bancos de hueso en donde poder preservar partes de calota craneana después de craneotomías descompresivas. La técnica de marsupialización consiste en realizar una incisión adicional a nivel abdominal y en almacenar el hueso en el tejido graso de la zona, pero resulta evidentemente una técnica invasiva en un paciente grave frente a otras medidas alternas, tales como la esterilización del fragmento óseo en óxido de etileno (4). Además, cuando el hueso permanece por tiempo prolongado en el área (marsupialización), sufre reabsorción en grado variable, que hace que su ajuste y resultado estético posterior sea subóptimo. Por estos motivos, la esterilización del fragmento óseo en oxido de etileno parece una alternativa económicamente viable para hospitales que carecen de bancos de hueso. Ningún paciente de nuestra serie a los que se les ha recolocado hueso tratado en esta manera ha presentado infección o rechazo del material.

Los materiales de osteosíntesis empleados para reconstrucción craneofacial iniciaron su historia clínica con la utilización de alambre, ya que era el único método que se conocía para poder estabilizar un trazo de fractura (7). En el año 1952, Leventhal (1) inició la era del titanio implantándolo en tejido subcutáneo de conejos y en el fémur de ratas, describiendo la formación a su alrededor de una cápsula tipo sinovial con ausencia de infiltración leucocitaria. No fue hasta el año 1977 cuando Branemark (3) publicó su trabajo sobre implantes de titanio en pacientes edéntulos y la osteointegración de este metal. Posteriormente se realizaron estudios sobre osteointegración de implantes de titanio en marco orbitario (8). Dada la falta de estabilidad tridimensional proporcionada por los alambres y las suturas en la reconstrucción craneofacial (9), en 1991 Sadove introdujo la utilización de placas y tornillos metálicos de pequeño calibre con el propósito de brindar una mayor estabilidad a las nuevas configuraciones óseas (10). Eppley (9), publicó la seguridad del uso de placas absorbibles y mallas compuestas de Ácido Láctico y Ácido Poliglicólico, con resultados muy similares a los obtenidos con titanio respecto a tasas de infección que fueron del $0.4 \%$.

La mayor ventaja del titanio sobre cualquier otro tipo de material aloplástico y en algunos casos del mismo hueso autólogo que puede presentar absorción aleatoria (11), es que el titanio tiene la capacidad de poder soportar grandes cargas bacterianas de 8 ó 12, así como más de 300 diferentes tipos de bacterias propias de la cavidad oral (6). Una de las grandes desventajas de cualquier material aloplástico colocado en cualquier área del cuerpo es la presencia de la denominada interfase fibrosa o cápsula (12), compuesta por un infiltrado leucocitario que puede promover la aparición de infecciones (6). En Cirugía Craneofacial la tasa de infecciones varía entre el 2.5 y el $6.5 \%(13,14)$. El titanio carece de esta interfase fibrosa cuando se implanta tanto en el tejido subcutáneo (1), como en el hueso (3) y, en casos complejos en los que se compromete el tejido respiratorio, ya sea por exposición de senos paranasales o de la cavidad faríngea, se ha comprobado que el titanio tiene una incorporación celular que se inicia como metaplasia escamosa, seguida de células glandulares y epitelio ciliar a los 3 meses. Por lo tanto, podemos estar seguros de que ya sea a nivel óseo por medio de osteointegración (3), así como incorporación de epitelio ciliar en el tracto respiratorio (6), este metal con características químicas similares al hueso, debe ser considerado como el Alejandro Magno en lo que respecta a su capacidad de invadir territorios hostiles y conquistarlos.

En cuanto al empleo de materiales plásticos, el que hemos empleado resulta de una mezcla de Polimetilmetacrilato y Polihidroximetilmetacrilato con sulfato de calcio y consta de un 30-40\% de porosidad en su arquitectura, lo que permite su fácil incorporación al hueso (Fig. 4). Estos materiales se han venido utilizando en Odontología desde hace 30 años para reemplazamiento de fosa dentaria (5); en Neurocirugía, otorgan la fortaleza de la que carecen las placas tradicionales de titanio de $0.6 \mathrm{~mm}$ de espesor y ya que se pueden emplear para una reconstrucción con un modelo computarizado en 3-D, confieren un resultado estético insuperable por la técnica de moldeado manual tradicional (15).

Cuando afrontamos la corrección de importantes lesiones óseas craneofaciales, independientemente de cuál sea su origen: traumatológico, tumoral o congénito, tienen que ser tratadas bajo los mismos principios básicos; al igual que existe un A,B,C en el manejo del paciente politraumatizado, en Cirugía Craneofacial estos principios deben ser: A. exposición adecuada del área comprometida; B, reducción precisa de la fractura más utilización de material de osteosíntesis con injertos autólogos y/o aloplásticos; C, impecable cobertura con tejidos blandos. Estos principios básicos han sido desde siempre la base fundamental de la Cirugía Craneofacial y están descritos ampliamente en múltiples trabajos $(2,7,16,17)$. 


\section{Exposición adecuada de área comprometida:}

La base fundamental es la disección subperióstica, concepto preconizado por Jean Paul Tessier $(16,17)$, por medio de abordajes bicoronal, intraoral, transconjuntival o subciliar. El instrumento de preferencia es el disector Freer.

\section{Precisa reducción de la fractura más utilización de material de osteosíntesis e injertos autólogos y/o aloplásticos:}

Este paso no se puede lograr sin el cumplimiento del enunciado anterior. Para la osteosíntesis se han utilizado alambres (7), titanio (10) y placas reabsorbibles de Ácido poliláctico y Ácido poliglicólico con el propósito de evitar la migración intracraneana del titanio en pacientes pediátricos; de darse el caso, el material debe ser retirado $(18,19)$. Más recientemente se ha implementado la utilización de segmentos óseos tridimensionales de polimetilmetacrilato poroso, que se adecuan como llave a cerradura a la medida del defecto del cráneo del paciente utilizando como base la TC (5). Todos estos métodos buscan el mismo propósito, la reducción precisa de las fracturas.

\section{Impecable cobertura con tejidos blandos:}

Lamentablemente, por muy bien que hayamos realizado los dos pasos anteriores, si no tenemos una adecuada cobertura de nuestros implantes autólogos o aloplásticos por tejidos blandos (20), vamos a tener una probabilidad muy alta de que éstos se expongan. Una de las formas de prevenir exposiciones, radica en una precisa resección de tejido desvitalizado en casos de traumatismos, evitar de cualquier manera el cierre a tensión de heridas operatorias y utilizar dos planos quirúrgicos para el cierre de incisiones.

\section{Conclusiones}

A pesar de que el número de pacientes de nuestro estudio es reducido, hemos pretendido ejemplificar las múltiples alternativas de reconstrucción craneofacial que pueden ser utilizadas en este tipo de patologías para solventar de manera satisfactoria y con tasas bajas de complicación lesiones complejas y potencialmente mortales por infección secundaria del sistema nervioso central.

Independientemente del tipo de material a utilizar para una reconstrucción craneofacial compleja, el factor más importante a tener en cuenta es el desarrollo de los principios básicos de la Cirugía Craneofacial: A, adecuada exposición subperióstica; $\mathrm{B}$, precisa reducción más fijación con material de osteosíntesis y C, impecable cobertura con tejidos blandos.
El trabajo conjunto de equipos quirúrgicos multidisciplinarios es la piedra angular para la obtención de resultados óptimos en la reconstrucción de lesiones craneofaciales complejas.

\section{Dirección del autor}

Dr. Gerardo Flores-Lima

Centro Panamericano de Ojos 91

Avenida Norte número 541

Colonia Escalón 2do Nivel.

e-mail: clinica@doctorgerardoflores.com

\section{Bibliografía}

1. Leventhal, G. S.: "Titanium, a metal for surgery". J. BoneJoint Surg. (Am.), 1951, 33: 473 .

2. Tessier P: "The definite plastic surgical treatment of severe facial deformities of craniofacial dysostoses, Crouzon's Apert's disease". Plast Reconstr Surg 1971, 48:419.

3. Branemark, P. I., Hansson, B. O., Adell, R., et al. "Osseointegrated implants in the treatment of the edentulous jaw: Experience from a 10-year period” Scand. J. Plast. Reconstr. Surg. Suppl. 1977, 16: 1 .

4. Jho DH, Neckrysh S.: " Ethylene oxide gas sterilization: A simple technique for storing explanted skull bone”. J Neurosurg 2007, 107: 440.

5. Eppley BL.: "Biomechanical testing of alloplastic PMMA cranioplasty materials". J Craniofac Surg., $2005,16(1): 140$.

6. Schubert W, Gear AJL, Lee Ch.: "Incorporation of Titanium Mesh in Orbital and Midface Reconstruction". Plast. Reconstr. Surg., 2002, 110: 1022

7. Wolfe, SA: "An examination of posttraumatic, postsurgical orbital deformities: Conclusions drawn for improvement of primary treatment". Plast. Reconstr. Surg 2008, 122:1870.

8. Allison, R. T., and Sugar, A. W.: "Production of high quality ground sections of bone containing metal implants to demonstrate osseo-integration: A simplified method". Med. Lab. Sci., 1990, 47: 168

9. Eppley BL, Morales L.: "Resorbable PLLA-PGA plate and screw fixation in pediatric craniofacial surgery: clinical experience in 1883 patients". Plast Reconstr Surg. 2004,114(4):850.

10. Sadove, A. M., and Eppley, B. L.: "Microfixation techniques in pediatric craniomaxillofacial surgery". Ann.Plast. Surg. 1991, 27: 36.

11. Tong, L., and Buchman, S. R: "Facial bone grafts: Contemporary science and thought". J. Craniomaxillofac. Trauma.2000, 6: 31.

12. Shapiro, M.A.: "Smooth vs. rough: An 8-year survey of mammary prostheses". Plast. Reconstr. Surg. , 1989, 84: 449.

13. Whitaker, L. A., Munro, I. R., Salyer, K. E., et al.: "Combined report of problems and complications in 793 craniofacial operations". Plast. Reconstr. Surg., 1979, 64: 198

14. Fearon, J. A., Bartlett, S. A., Munro, I. R., and Whitaker, L.: "Infection in craniofacial surgery: A combined report of 567 procedures from two centers". Plast. Reconstr.Surg., 1997, 100: 862.

15. Marsh JL, Vannier MW: "The "third" dimension in craniofacial surgery”. Plast Reconstr Surg, 1983, 71(6):759.

16. Jones, B M.: "Paul Louis Tessier", Plast. Reconstr. Surg., 2008,122(4):1297.

17. Wolfe, S A.: "Paul Louis Tessier, M.D., 1917 to 2008". Plast. Reconstr. Surg. 2008, 122(4): 1294.

18. Orringer, J. S., Barcelona, V., and Buchman, S. R.: "Reasons for removal of rigid internal fixation devices in craniofacial surgery". J. Craniofac. Surg., 1998, 9: 40

19. Eppley, B. L., Sadove, A. M., and Havlik, R. J.: "Resorbable plate fixation in pediatric craniofacial surgery".Plast. Reconstr. Surg., 1997, 100: 1 .

20. Baumeister, S, Peek, A, Friedman, A.: "Management of postneurosurgical bone flap loss cause by infection”. Plas. Reconstr. Surg. 2008,122(6): $195 \mathrm{e}$ 